\title{
The search for Lepton-Flavour Violation with the Mu3e Experiment
}

\author{
F. Wauters* for the Mu3e Collaboration \\ Johannes Gutenberg University Mainz. \\ E-mail: fwauters@uni-mainz.de
}

The Mu3e experiment aims to measure the lepton-flavour violating decay $\mu^{+} \rightarrow e^{+} e^{+} e^{-}$at the $2 \cdot 10^{-16}$ level, initially with a single event sensitivity of $2 \cdot 10^{-15}$ for the phase I detector described here. A DC muon beam at the Paul Scherrer Institute (PSI) of $10^{8} \mu^{+} / \mathrm{s}$ will be stopped inside a Si pixel tracker constructed from High-Voltage Monolithic Active Pixel Sensors (HV-MAPS), complemented with timing detectors. An excellent momentum resolution is achieved by tracking the low momentum positrons (electrons) inside a $1 \mathrm{~T}$ magnetic field. With multiple scattering being the limiting factor on the momentum resolution, each detector layer has a thickness of approximately $0.1 \%$ of a radiation length. Currently, all detector prototypes perform according to the required specifications, and their design is being finalized. The collaboration is working to integrate all detector systems and install the experiment at PSI.

The 20th International Workshop on Neutrinos (NuFact2018)

12-18 August 2018

Blacksburg, Virginia

${ }^{*}$ Speaker. 


\section{The Mu3e Experiment}

Searching for Charged Lepton Flavour Violation (CLFV) in muon decays is a remarkabally sensitive method to search for new physics. The CLFV decays are free from Standard Model backgrounds, with a relatively simple and clear signature in the detectors. In addition, modern proton accelerator facilities provide a high yield of muons as a secondary beam. The Mu3e experiment is part of ongoing global effort searching for CLFV in muon decays [1, 2, 3, 4, 5], aiming for a single event sensitivity of $2 \cdot 10^{-15}$ on the process $\mu^{+} \rightarrow e^{+} e^{+} e^{-}$, an improvement of 3 orders of magnitude over the SINDRUM result [6].

\subsection{The Mu3e detector}

The Mu3e experiment will run at the Paul Scherrer Institute, where $10^{8} \mu^{+} / s$ available at the compact muon beam line will be stopped on a double-coned mylar target. At its heart, the Mu3e detector is a cylindrical Si pixel tracker sitting in a $1 \mathrm{~T}$ magnetic field (figure 1). For signal events, 2 positron tracks and 1 electron track share a common vertex, have a balanced momentum and a summed energy equal to the muon mass. The main backgrounds are internal conversion decays, $\mu \rightarrow e e e v v$, and Bhabha scattered positrons combined with a Michel positron from a second muon decay. An excellent momentum resolution better then $1 \mathrm{MeV} / \mathrm{c}$ is required to separate signal from background. For this reason, we have developed HV-MAPS [7] which are thinned to $50 \mu \mathrm{m}$ to minimize multiple scattering. Combined with a Kapton support structure, ducts to guide helium cooling gas, and low-Z flex print cables for signal, control, and power, one detector layer has a thickness of $\approx 0.1 \%$ of a radiation length. To sufficiently suppress combinatorial backgrounds, additional timing detectors are added: 4 layers of scintillating fibers between the inner and outer central detector, and a scintillating tile detector at the outer detector stations.

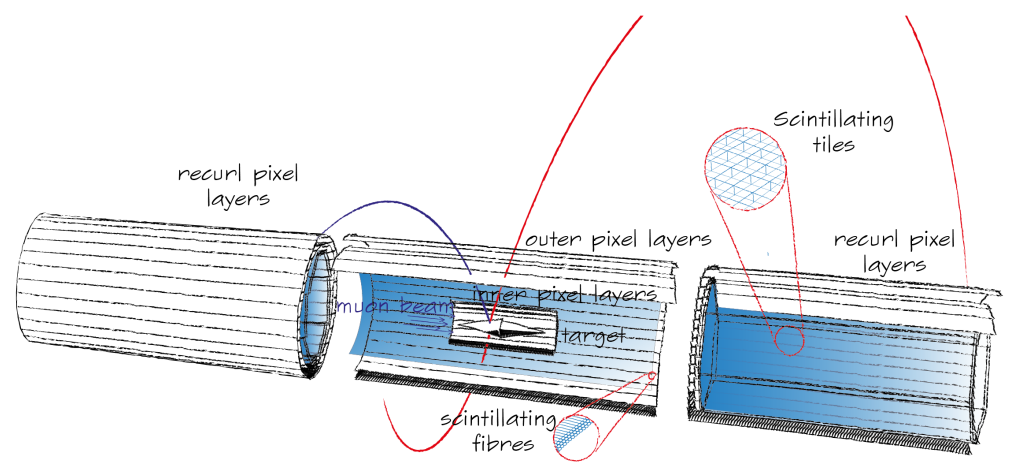

Figure 1: The Mu3e detector. A Si pixel tracker achieves optimal momentum resolution by letting the Michel electrons/positrons recurl in a homogeneous magnetic field. An inner central detector close to the stopping target allows for good vertex resolution.

\subsection{Data Acquisition System and Reconstruction}

To identify candidate signal events, three electron/positron tracks need to be reconstructed. The full detector information needs to be read out triggerless before a decision can be taken. 114 Front-end FPGA boards inside the magnet collect the LVDS serial data from the detector ASICs, 
and send it off via optical fibres to the filter farm at a data rate of about $100 \mathrm{Gbit} / \mathrm{s}$. There, the data gets distributed to 12 FGPA+GPU boxes, each machine receiving time slices containing all detector hits. On the GPUs, the events are reconstructed online and selected. Selected data frames get written to disk with a reduction factor $\sim 100$. We showed that with commercial GPUs available today, and an optimized reconstruction algorithm [8], this reduction factor can be achieved with a 98\% reconstruction efficiency [9] for signal events in the acceptance of the detector.

\section{Status of the project}

\subsection{The pixel tracker and timing detector}

MuPix7, our small 32x40 pixel matrix 3x3 mm chip which meets all further requirements for the experiment, was extensively tested during 2016 and 2017 and showed good performance [10, 11]. Our latest nearly full size prototype, the $1 \times 2 \mathrm{~cm}$ MuPix 8 chip, is currently under evaluation, and shows promising results with efficiencies $>99.5 \%$ and a time resolution of $<15 \mathrm{~ns}$ (figure 2). The final chip will be $2 \times 2 \mathrm{~cm}$. Detector modules are made up from strips of 6 to 18 MuPix ASICs. The central and outer trackers combined will contain 2844 chips, each with a $1.25 \mathrm{Gbit} / \mathrm{s}$ link to the front-end electronics. Thermo-mechanical and cabling mockups are being constructed to test the mechanical design of the detector.

Recent test-beam results show that both the scintillating fiber and the tile detector prototypes meet all requirements, with a respective time resolution of $350 \mathrm{ps}$ and $70 \mathrm{ps}$. There SiPM signals are being read out by a custom ASIC called MuTrig [12]. The $1.25 \mathrm{Gbit} / \mathrm{s}$ data from these ASICs is combined with the pixel data for further online processing.

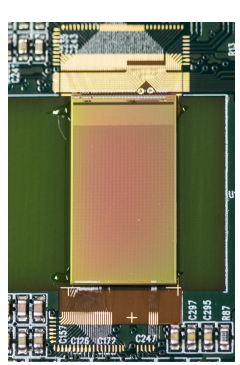

(a)

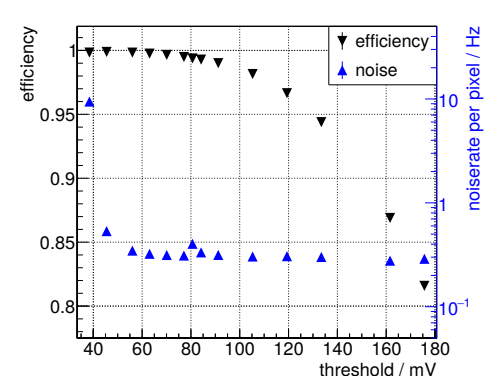

(b)

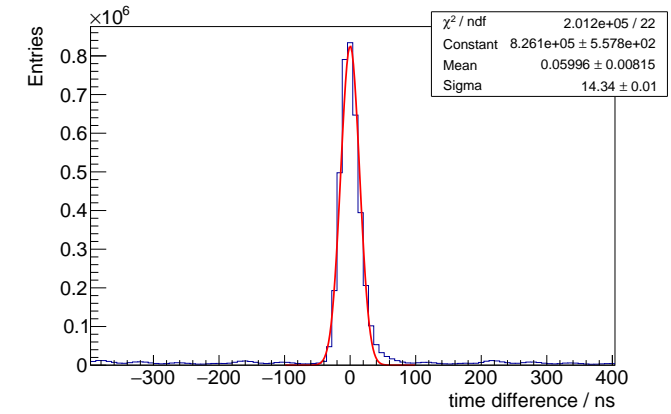

(c)

Figure 2: The a) $1 \times 2 \mathrm{~cm}$ large MuPix 8 chip [13]: Efficient charge collection allows for a wide range of working points with b) optimal efficiency and a low noise figure, and c) a time resolution of $<15 \mathrm{~ns}$.

\subsection{Detector construction and commissioning}

Since 2018 we have a full mechanical design of the experiment, and the collaboration is working towards a full integration of all detector systems. Test beam campaigns are conducted with a vertical slice of the data acquisition, containing prototypes of the FPGA readout boards, reading both timing and pixel detectors. At PSI, where the experimental area is being prepared to host the 
experiment, our 1T magnet will be delivered in 2019. We are expecting to start commissioning the system in 2020 once the first detector modules are constructed. Once data taking starts, the target single event sensitivity of phase I Mu3e of $2 \cdot 10^{-15}$ will be reached after 300 days of data taking.

\section{References}

[1] MEG collaboration, T. Mori, Final Results of the MEG Experiment, Nuovo Cim. C39 (2017) 325 [1606.08168].

[2] C. Voena, Status and prospects of charged lepton flavor violation searches with the MEG-II experiment, PoS NuFact2017 (2017) 104.

[3] DeEME collaboration, N. Teshima, DeeMe experiment to search for muon to electron conversion at J-PARC MLF, POS NuFact2017 (2018) 109.

[4] COMET collaboration, M. Lee, COMET muon conversion experiment in J-PARC, PoS NuFact2017 (2018) 107.

[5] S. Giovannella, Status of the Mu2e experiment, EPJ Web Conf. 179 (2018) 01003.

[6] SINDRUM collaboration, U. Bellgardt et al., Search for the Decay $\mu^{+} \longrightarrow e^{+} e^{+} e^{-}$, Nucl.Phys. B299 (1988) 1.

[7] I. Peric, A novel monolithic pixelated particle detector implemented in high-voltage CMOS technology, Nucl.Instrum.Meth. A582 (2007) 876.

[8] Kozlinskiy, Alexandr, Track reconstruction for the Mu3e experiment based on a novel multiple scattering fit, EPJ Web Conf. 150 (2017) 00005.

[9] vom Bruch, Dorothea, Online data reduction using track and vertex reconstruction on GPUs for the Muзe experiment, EPJ Web Conf. 150 (2017) 00013.

[10] H. Augustin et al., Efficiency and timing performance of the MuPix7 high-voltage monolithic active pixel sensor, Nucl. Instrum. Meth. A902 (2018) 158 [1803.01581].

[11] H. Augustin, N. Berger, S. Dittmeier, C. Grzesik, J. Hammerich, Q. Huang et al., The MuPix telescope: A thin, high-rate tracking telescope, Journal of Instrumentation 12 (2017) C01087.

[12] H. Chen, W. Shen, K. Briggl, V. Stankova, Y. Munwes, D. Schimansky et al., Characterization Measurement Results of MuTRiG - A Silicon Photomultiplier Readout ASIC with High Timing Precision and High Event Rate Capability, PoS TWEPP-17 (2017) 008.

[13] H. Augustin, N. Berger, S. Dittmeier, F. Ehrler, C. Grzesik, J. Hammerich et al., MuPix8: A large area monolithic HVCMOS pixel detector for the mu3e experiment, Nuclear Instruments and Methods in Physics Research Section A: Accelerators, Spectrometers, Detectors and Associated Equipment (2018) . 